\title{
Comparison of Seropositivity to Trichomonas vaginalis between Men with Prostatic Tumor and Normal Men
}

\author{
Jung-Hyun Kim¹, Hong-Sang Moon², Kyu-Shik Kim², Hwan-Sik Hwang³, Jae-Sook Ryu', Sung-Yul Park²,* \\ ${ }^{1}$ Department of Environmental Biology and Medical Parasitology, ${ }^{2}$ Department of Urology, ${ }^{3}$ Department of Family Medicine, Hanyang University \\ College of Medicine, Seoul 04763, Korea
}

\begin{abstract}
Trichomoniasis is the most common curable sexually-transmitted infection. Most Trichomonas vaginalis-infected men are asymptomatic and can remain undiagnosed and untreated, and this has been thought to result in chronic persistent prostatic infection. Chronic inflammation is regarded as the major factor in the pathogenesis and progression of benign prostatic hyperplasia $(\mathrm{BPH})$ and prostatic cancer $(\mathrm{PCa})$. The aim of this study is to identify seropositivity to $T$. vaginalis in men with prostate tumors (BPH or PCa) visited to Hanyang University Hospital. A total of 183 men were enrolled between October 2013 and November 2014. They consisted of 139 with BPH (mean age: 64.0 \pm 0.07 ) and 44 with prostate cancer (mean age: $73.3 \pm 0.18$ ). We carried out ELISA to identify the seropositivity to T. vaginalis. Mixed lysate antigen extracted from 8 strains of $T$. vaginalis was used in the ELISA. Also 58 male outpatients visited to Health Promotion Center in Hanyang University Hospital were evaluated for comparing group. As a results, seropositivity to $T$. vaginalis in patients with prostatic diseases was $19.7 \%$ (BPH: 18.7\%, PCa: $22.7 \%$ ) and it was significantly higher than the $1.7 \%$ of the comparing healthy group $(P=0.001)$. Therefore, prostatic tumor showed higher seropositivity against $T$. vaginalis than normal men. As far as we know, this is the first report about seroprevalence in prostatic tumor in Korea.
\end{abstract}

Key words: Trichomonas vaginalis, seropositivity, benign prostatic hyperplasia, prostate cancer

The prostate gland is believed to serve as a parasite reservoir in Trichomonas vaginalis infection in men [1]. Recently prostate cancer (PCa) is growing rapidly, accounting for a high proportion of male cancer cases. Usually the occurrence of cancer is often related with multiple factors. There is an increasing report of relevance to inflammation as a factor causing cancer reaction.

Trichomonas vaginalis had been found in the prostate parenchyma as well as the mucosa of the urethra and prostatic duct [1]. T. vaginalis is generally known as a surface-dwelling organism but the discovery of trichomonads in endo-epithelial cells and in sub-epithelial tissues has shown that they are capable of invasion [1,2]. Chronic inflammation is known to play an important role at the occurrence and progression of several types of cancer, including prostate cancer [3]. There are many reports that chronic inflammations have associated with benign prostatic hyperplasia (BPH)/PCa. It also reported that chronic inflammation of benign prostate tissue was associated with PCa [4].

\footnotetext{
- Received 1 January 2019, revised 8 February 2019, accepted 10 February 2019.

*Corresponding author (syparkuro@hanyang.ac.kr)

(C) 2019, Korean Society for Parasitology and Tropical Medicine

This is an Open Access article distributed under the terms of the Creative Commons

Attribution Non-Commercial License (http://creativecommons.org/licenses/by-nc/4.0) which permits unrestricted non-commercial use, distribution, and reproduction in any

medium, provided the original work is properly cited.
}

Trichomoniasis is the most common curable sexually-transmitted infection, with an estimated 276.4 million new cases worldwide annually [5]. The rate of infection of male partners by women with trichomonal vaginitis is reported to reach $73 \%$ [6]. Over $75 \%$ of men harboring T. vaginalis are asymptomatic and may not seek treatment, and this can result in chronic inflammation [7].

Prostatitis is a very common disease with high prevalence rate ranging from 3-16\%, and half of patients had a recurrence after appropriate treatment. Half of the male may have prostatitis once in a lifetime. Prostatitis can occur in all ages and is known as the most common outpatient disease in urology clinic, especially in the more than 50-year-old man. One hundred fifty one patients (10.5\%) were reported to detect with trichomonas infection in the 1,442 patients with chronic prostatitis in Croatia men [8]. Also, high infection rate (34\%) of trichomonas infection by PCR was found in tissues of BPH [9]. So, $T$. vaginalis was recognized as a major cause of chronic prostatitis and $\mathrm{BPH}$.

However, there are a few reports on the prevalence of trichomonas infection in Korean men. Lee et al. [10] reported a higher rate of T. vaginalis infection (21.2\%) in patients with chronic prostatitis and urethritis with PCR method. In recent 
study, the prevalence of T. vaginalis and associated clinical conditions were evaluated in male patients from a primary care urology clinic in South Korea. Among the T. vaginalis positive patients, $87.5 \%$ had prostatic diseases, such as prostatitis and BPH [11].

Sutcliffe et al. [12] firstly reported that a group of PCa had a higher seropositive rate to T. vaginalis (12.6\%) than controls (9.4\%). Stark et al. [13] also support for an association between a seropositive status for antibodies against $T$. vaginalis and the risk of Pca by analysis of large prospective study. Nontheless, the studies for seropositivity to T. vaginalis in prostatic tumor (BPH and $\mathrm{PCa}$ ) as compared to the control group were not many in the world. No study has been reported in Korea. The purpose of this study is to investigate the serum antibodies to T. vaginalis in $\mathrm{BPH}$ and $\mathrm{PCa}$ patients and to compare with control healthy group.

As study population, a total of 183 men were enrolled between October 2013 and November 2014. They consisted of 139 with BPH (mean age: $64.0 \pm 0.07$ ) and 44 with prostate cancer (mean age: $73.3 \pm 0.18$ ) who were undergoing treatment in the Department of Urology, Hanyang University Seoul \& Guri Hospital. Inclusion criteria of BPH were (1) scores of 8 and more as International prostate symptom scores (IPSS) (2) $<4$ $\mathrm{ng} / \mathrm{ml}$ of PSA (3) $>20 \mathrm{ml}$ of prostate volume (measured by transrectal ultrasonography; TRUS), and (4) $>4 \mathrm{ng} / \mathrm{ml}$ of PSA and no evidence of malignant tumor on TRUS biopsy. Also, PCa patients proven by TRUS biopsy were only selected if they had undergone radiologic evaluation to determine their TNM clinical stage. Radiologic evaluation was carried out via prostate MRI and whole body bone scan (Table 1).

Sera were obtained from 139 patients with BPH, 44 with prostate cancer and 58 male healthy volunteers (mean age: $40.4 \pm 0.15$ ) who visited Hanyang University International Hospital Health Promotion Center. Healthy volunteers had no history of prostatic disease [14]. Blood samples of $7 \mathrm{ml}$ were collected, and plasma was stored at $-70^{\circ} \mathrm{C}$. This study was ap- proved by the Institutional Review Board of Hanyang University and written informed consent was obtained from patients and controls (IRB No. HYUH 2013-04-028-006). For determining the sensitivities of the ELISA assay, 52 sera from women with trichomoniasis who visited the Gynecology Clinic formed the positive control group. For the negative control group, sera were collected from 27 male volunteers aged between 21 and 24 years with no history of any sexual encounters.

For antigen preparation of ELISA, 8 T. vaginalis strains were used for the mixed lysate Ag (1 Korean strain [KT4], 2 Korean strains from men [KT55, KT56], 2 metronidazole-resistant Korean strains [KT52, KT53], 1 metronidazole sensitive [T016] and 1 resistant [IR78] USA strain, and 1 metronidazole sensitive Japan strain [YA-1]).

Each of 8 strains was cultivated in TYM (trypticase-yeast extract-maltose) medium supplemented with 10\% horse serum, washed 3 times with PBS and frozen at $-70^{\circ} \mathrm{C}$ and thawed at $4^{\circ} \mathrm{C}[15]$. And then, it was disrupted by sonication. After centrifugation at 10,000 $\mathrm{g}$ for $1 \mathrm{hr}$, the supernatant was stored at $-70^{\circ} \mathrm{C}$. For the ELISA experiment, 96-well ELISA plates were coated with $5 \mu \mathrm{g} / \mathrm{ml}$ of diluted mixed lysate Ag and incubated overnight at $4^{\circ} \mathrm{C}$. The plates were washed thrice with PBSTween 20 (0.05\% Tween 20 in PBS [pH 7.0]) followed by the addition of $100 \mu \mathrm{l} /$ well of blocking buffer (5\% BSA [bovine serum albumin] in coating buffer). After incubation at $37^{\circ} \mathrm{C}$ for $2 \mathrm{hr}$, the plates were washed thrice with PBS-Tween 20. Serum diluted 1:100 in PBS containing 2\% BSA, was added to each well. After incubation for $1 \mathrm{hr}$ at $37^{\circ} \mathrm{C}$, the plates were washed thrice with PBS-Tween 20, followed by the addition of $100 \mu \mathrm{l} /$ well of secondary goat anti-human IgG (Jackson Immuno Research, West Grove, Pennsylvania, USA) conjugated to horseradish peroxidase and diluted 1:40,000 in PBS containing $2 \%$ BSA. The plates were incubated for $1 \mathrm{hr}$ at $37^{\circ} \mathrm{C}$ and washed thrice with PBS-Tween 20. Color was developed by adding $100 \mu \mathrm{l} /$ well of substrate solution (TMB Microwell

Table 1. Patient demographic and clinical data

\begin{tabular}{|c|c|c|c|c|}
\hline & No disease & $\mathrm{BPH}$ & $\mathrm{PCa}$ & $\mathrm{BPH}+\mathrm{PCa}$ \\
\hline Total number & 58 & 139 & 44 & 183 \\
\hline Mean age & $40.4 \pm 0.15$ & $64.0 \pm 0.07$ & $73.3 \pm 0.18$ & $66.2 \pm 0.06$ \\
\hline PSA (ng/ml) & $0.9 \pm 0.00$ & $2.6 \pm 0.03$ & $90.2 \pm 5.75$ & $23.9 \pm 0.71$ \\
\hline TRUS (ml) & - & $39.3 \pm 0.13$ & $52.2 \pm 0.98$ & $41.9 \pm 0.14$ \\
\hline
\end{tabular}

All the data are expressed as means \pm standard errors except for total numbers.

$\mathrm{BPH}$, benign prostatic hyperplasia; PCa, prostate cancer; PSA, prostate-specific antigen; TRUS, transrectal ultrasonography. 
Peroxidase Substrate System, KPL, Gaithersburg, Maryland, USA) and incubating the plates at room temperature for 30 min. Absorbance was measured with an ELISA reader (BioRad, Hercules, California, USA) at $490 \mathrm{~nm}$ after 20 min incubation with stop solution $\left(2.5 \mathrm{M} \mathrm{H}_{2} \mathrm{SO}_{4}\right)$. Sera were classified as negative if the ELISA ODs were lower than the relevant cutoff value. These were established as the mean OD (0.143) plus twice the standard deviation ODs (0.0284) in samples obtained from the 27 healthy male volunteers. The cutoff OD value measured was 0.2 . Serosensitivity (71.2\%) in 52 women infected with T. vaginalis was significantly higher than that of 27 male healthy control (3.7\%). Statistical analysis was performed using SPSS 21.0 software (IBM, Armonk, New York, USA). The results were compared using Chi-square tests.

As results, serologic positive rate of $139 \mathrm{BPH}$ and $44 \mathrm{PCa}$ were $18.7 \%$ and $22.7 \%$, respectively. Statistical difference was not observed between 2 groups. Each group had significantly increased seropositivity compared with that of comparing group $(\mathrm{n}=58)$ who visited Hanyang University International Hospital Health Promotion Center and had no history of prostatic disease (1.7\%). Also, the positive rate (19.7\%) of 183 prostatic disease (139 $\mathrm{BPH}+44 \mathrm{PCa}$ ) showed significantly increased seropositivity than that of comparing group $(P=0.001)$ (Fig. 1). The optical densities of women trichomoniasis seemed to be more widely distributed than those of prostatic disease (data not shown). Also, prevalence of patients in Seoul and Guri Hospital showed significant differences as 3.8\%, $31.4 \%$, respectively $(P$-value $=0.000)$. There is a regional difference of seropositivity to T. vaginalis (data not shown).

Prostatic inflammation plays a central role in the patho- physiology of prostatic disease, as chronic inflammation is often found in biopsy and surgical specimens of men with $\mathrm{BPH} /$ PCa. One possible source of chronic, prostatic inflammation is sexually transmitted infections. T. vaginalis infection is the most common curable sexually transmitted infection in worldwide, and it can elicit chronic inflammation within the parenchyma of prostatic glands.

The epidemiologic evidence for an association of T. vaginalis and PCa risk showed conflicting results as follows; Sutcliffe et al. [12] and Stark et al. [13] suggested association between seropositivity to T. vaginalis and the risk of PCa. However, Shui et al. [16] examined that $T$. vaginalis serostatus was not associated with an increased risk of metastatic PCa.

The aim of this study was to assess whether BPH/PCa is associated with T. vaginalis serostatus in Korea. Serologic positive rate of $139 \mathrm{BPH}$ and $44 \mathrm{PCa}$ were $18.7 \%$ and $22.7 \%$, respectively. In contrast, healthy control showed low serologic evidence of $1.7 \%$. Thus, it is suggested that about $20 \%$ of BPH/ PCa patients are presumed to have T. vaginalis infection in their infection history. However, higher seropositivity in BPH and $\mathrm{PCa}$ is partly explained by their old age (mean age: $\mathrm{BPH}$; 64.0, PCa; 73.3 years) comparing with younger age of healthy group (40.4 years).

Trichomoniasis is a sexually transmitted infection. Thus, theoretically one isolate could spread around the world. But various biological characteristics such as virulence level or metronidazole sensitivity level of T. vaginalis, isolates from male or female, isolates from domestic or foreign country, could affect on the antigenicity of T. vaginalis. Our previous report, mixed lysate antigen of 6 strains showed higher sensitivity than that
A

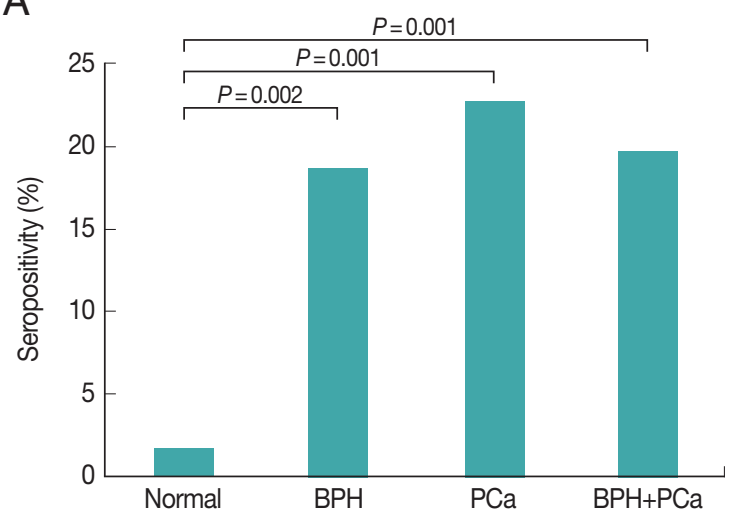

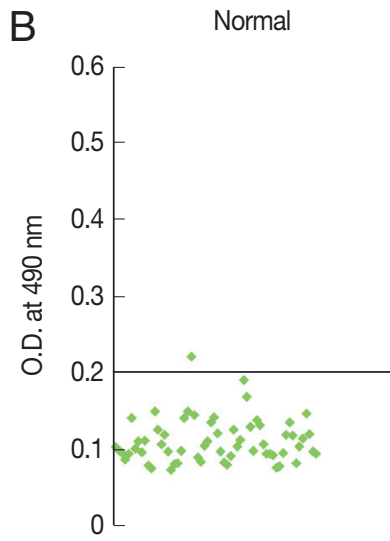

$\mathrm{BPH}+\mathrm{PCa}$

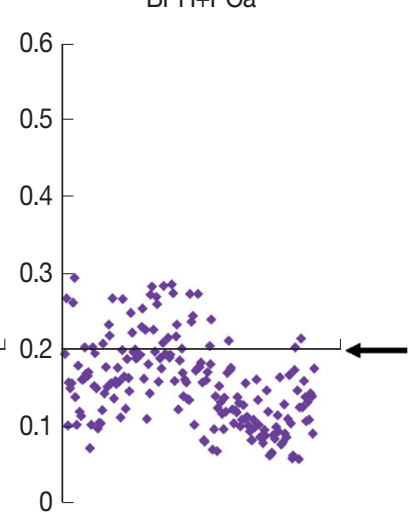

Fig. 1. Seropositivity to Trichomonas vaginalis in men with prostatic tumor. To compare for seropositivity of $T$. vaginalis, sera were obtained from 183 patients with prostatic diseases (44 PCa, $139 \mathrm{BPH}$ ) and 58 normal men. (A) Antibody positivity of the sera to T. vaginalis. (B) Antibody titers of the sera. Arrow indicates a cutoff value. 
of single strain as antigen in ELISA [15]. Also, mixed lysate antigen from 8 strains showed higher sensitivity and specificity than a-actinin [17]. Therefore, in this study, mixed lysate antigen is prepared with 8 strains of T. vaginalis. Two USA, 1 Japan and 5 Korean strains were included in mixed antigen. Also, 2 Korean strains from men and 3 metronidazole-resistant strains were contained in mixed antigen. The sensitivity and specificity of this ELISA was $71.2 \%$ and $96.3 \%$, respectively. Five studies which dealt with the performance of ELISAs for vaginal trichomoniasis have been reviewed; their pooled sensitivity was $82 \%$ and pooled specificity was $73 \%$ [18]. The ELISA using mixed lysate Ag in the present study demonstrated higher specificity (96.3\%) than the pooled data, while their sensitivity was similar to that of the pooled data.

Women trichomoniasis indicated widely distributed optical density (data not shown). In contrast, prostatic disease patients showed optical density of narrow range (Fig. 1). Therefore, it is necessary to develop ELISA to know exact seroprevalence of male trichomoniasis.

This study had several limitations. First limitation is the agedifference between the study group and the healthy control group. Actually, the prevalence of prostate disease in men over 60 is more than $60 \%$. It was not easy to get prostate-related healthy volunteers who were older than 60 . Second limitation is that the population number of this study (especially $\mathrm{PCa}$ ) was small $(\mathrm{n}=44)$. Sepositivity of PCa $(22.7 \%)$ is higher than that of BPH (18.7\%) though it was not marked difference between the 2 groups. In addition, $57 \mathrm{BPH}(1.8 \%)$ and $21 \mathrm{PCa}$ $(9.5 \%)$ in Seoul Hospital showed distinct difference in seroprevalence although $P$-value between 2 groups is 0.11 . Presumably based on this result, if a large-scale study would be conducted, the seropositive rate in PCa group may be expected to be higher than $\mathrm{BPH}$.

Interestingly, there is a regional difference in seropositivity to T. vaginalis between Seoul and Guri Hospital. Prevalences of patients in Seoul and Guri Hospital are 3.8\% and 31.4\%, respectively (data not shown). However, our previous studies have been reported higher prevalence of $T$. vaginalis in Guri city; 424 women attending an outpatient health screening at Guri Hospital showed higher seropositivity (39.2\%) by ELISA, and 177 symptomatic women showed a high positivity rate of $10.4 \%$ by PCR $[17,19]$. These high infection rates in women residing in Guri city would be helpful for explanation about high seropositivity in men of Guri city. More research to find out the cause of regional differences in seropositivity to T. vagi- nalis is required.

However, T. vaginalis had been found in prostate stroma and epithelium by immunoperoxidase stain, in BPH tissue and urine of prostatitis by PCR [1,2,9-11]. Our previous studies demonstrated that T. vaginalis experimentally cause inflammatory response in prostate epithelial and stromal cell lines through cytokine production and migration of inflammatory cells, and also induce proliferation of stromal cell via crosstalk with mast cell [20-22] In addition, Sutcliffe et al. [23] hypothesized that $T$. vaginalis infection may contribute to carcinogenesis via inflammation; T. vaginalis adherence to prostate epithelial cells trigger a cell-signaling cascade through known proto-oncogenes, PIM1, c-MYC, HMGA-1, that may ultimately lead to prostate carcinogenesis. In line with above description, to explore association between T. vaginalis and $\mathrm{PCa} / \mathrm{BPH}$, further study on seroprevalence with a large number of prostatic tumor $(\mathrm{PCa} / \mathrm{BPH})$ and age-matched control is required.

Taken together, the patients with prostatic tumor such as $\mathrm{BPH}$ and PCa showed significantly higher seropositivity to T. vaginalis than healthy group without prostatic diseases. This is the first report about seroprevalence in prostatic tumor in Korea.

\section{ACKNOWLEDGMENT}

This work was supported by the research fund of Hanyang University (HY-201200000002843).

\section{CONFLICT OF INTEREST}

The authors declared that they have no conflicts of interest.

\section{REFERENCES}

1. Gardner WA Jr, Culberson DE, Bennett BD. Trichomonas vaginalis in the prostate gland. Arch Pathol Lab Med 1986; 110: 430-432.

2. Gardner WA Jr, Culberson DE, Stafford JR. Subepithelial organisms in trichomonal cervicitis. Diagn Cytopathol 1987; 3: 227229.

3. Grivennikov SI, Greten FR, Karin M. Immunity, inflammation, and cancer. Cell 2010; 140: 883-899.

4. Gurel B, Lucia MS, Thompson IM Jr, Goodman PJ, Tangen CM, Kristal AR, Parnes HL, Hoque A, Lippman SM, Sutcliffe S, Peskoe SB, Drake CG, Nelson WG, De Marzo AM, Platz EA. Chronic inflammation in benign prostate tissue is associated with high-grade prostate cancer in the placebo arm of the prostate cancer prevention trial. Cancer Epidemiol Biomarkers Prev 2014; 23: $847-856$. 
5. World Health Organization. Global Incidence and Prevalence of Selected Curable Sexually Transmitted Infections-2008. Geneva, Switzerland. World Health Organization. 2012, pp 1-20.

6. Hobbs MM, Lapple DM, Lawing LF, Schwebke JR, Cohen MS, Swygard H, Atashili J, Leone PA, Miller WC, Seña AC. Methods for detection of Trichomonas vaginalis in the male partners of infected women: implications for control of trichomoniasis. J Clin Microbiol 2006; 44: 3994-3999.

7. Seña AC1, Miller WC, Hobbs MM, Schwebke JR, Leone PA, Swygard H, Atashili J, Cohen MS. Trichomonas vaginalis infection in male sexual partners: implications for diagnosis, treatment, and prevention. Clin Infect Dis 2007; 44: 13-22.

8. Skerk V, Krhen I, Schonwald S, Cajic V, Markovinovic L, Roglic S, Zekan S, Andracevic AT, Kruzic V. The role of unusual pathogens in prostatitis syndrome. Int J Antimicrob Agents 2004; 24: 5356.

9. Mitteregger D, Aberle SW, Makristathis A, Walochnik J, Brozek W, Marberger M, Kramer G. High detection rate of Trichomonas vaginalis in benign hyperplastic prostatic tissue. Med Microbiol Immunol 2012; 201: 113-116.

10. Lee JJ, Moon HS, Lee TY, Hwang HS, Ahn MH, Ryu JS. PCR for diagnosis of male Trichomonas vaginalis infection with chronic prostatitis and urethritis. Korean J Parasitol 2012; 50: 157-159.

11. Seo JH, Yang HW, Joo SY, Song SM, Lee YR, Ryu JS, Yoo, ES, Lee, WK, Kong, HH, Lee, SE, Lee, WJ, Goo YK, Chung DI, Hong Y. Prevalence of Trichomonas vaginalis by PCR in men attending a primary care urology clinic in South Korea. Korean J Parasitol 2014; 52: 551-555.

12. Sutcliffe S, Giovannucci E, Alderete JF, Chang TH, Gaydos CA, Zenilman JM, De Marzo AM, Willett WC, Platz EA. Plasma antibodies against Trichomonas vaginalis and subsequent risk of prostate cancer. Cancer Epidemiol Biomarkers Prev 2006; 15: 939945.

13. Stark JR, Judson G, Alderete JF, Mundodi V, Kucknoor AS, Giovannucci EL, Platz EA, Sutcliffe S, Fall K, Kurth T, Ma J, Stampfer MJ, Mucci LA. Prospective study of Trichomonas vagina- lis infection and prostate cancer incidence and mortality: physicians' health study. J Natl Cancer Inst 2009; 101: 1406-1411.

14. Kim KS, Moon HS, Park SY, Hwang HS, Kim JH, Kim SS, Han IH, Kim KJ, Noh CS, Ryu JS. Serum CCL11 levels in benign prostatic hyperplasia and prostate cancer. Urogenit Tract Infect 2016; 11: 103-108.

15. Ryu JS, Yoon K, Ha SE, Min DY, Ahn MH. Comparison of three Trichomonas antigens for the detection of IgG antibody in serum. Korean J Clin Microbiol 2000; 3: $62-68$ (in Korean).

16. Shui IM, Kolb S, Hanson C, Sutcliffe S, Rider JR, Stanford JL. Trichomonas vaginalis infection and risk of advanced prostate cancer. Prostate 2016; 76: 620-623.

17. Kim SR, Kim JH, Park SJ, Lee HY, Kim YS, Kim YM, Hong YC, Ryu JS. Comparison between mixed lysate antigen and a-actinin antigen in ELISA for serodiagnosis of trichomoniasis. Parasitol Int 2015; 64: 405-407.

18. Patel SR, Wiese W, Patel SC, Ohl C, Byrd JC, Estrada CA. Systematic review of diagnostic tests for vaginal trichomoniasis. Infect Dis Obstet Gynecol 2000; 8: 248-257.

19. Ryu JS, Chung HL, Min DY, Cho YH, Ro YS, Kim SR. Diagnosis of trichomoniasis by polymerase chain reaction. Yonsei Med J 1999; 40: 56-60.

20. Seo MY, Im SJ, Gu NY, Kim JH, Chung YH, Ahn MH, Ryu JS. Inflammatory response of prostate epithelial cells to stimulation by Trichomonas vaginalis. Prostate 2014; 74: 441-449.

21. Im SJ, Han IH, Kim JH, Gu NY, Seo MY, Chung YH, Ryu JS. Inflammatory response of a prostate stromal cell line induced by Trichomonas vaginalis. Parasite immunol 2016; 38: 218-227.

22. Kim JH, Kim SS, Han IH, Sim S, Ahn MH, Ryu JS. Proliferation of prostate stromal cell induced by benign prostatic hyperplasia epithelial cell stimulated with Trichomonas vaginalis via crosstalk with mast cell. Prostate 2016; 76: 1431-1444.

23. Sutcliffe S, Neace C, Magnuson NS, Reeves R, Alderete JF. Trichomonosis, a common curable STI, and prostate carcinogenesis--a proposed molecular mechanism. PLoS Pathog 2012; 8: e1002801. 
